\title{
The Interaction of Failure Modes in the Compression Response and Failure of Laminated Composites
}

\author{
Pavana Prabhakar, Anthony M Waas`and Ravi S. Raveendra ${ }^{\dagger}$
}

\begin{abstract}
Failure mode interactions when a laminated fiber reinforced composite is subjected to compression is studied here. Delamination, fiber kink-banding and their interaction are seen to dominate the failure response. An upscaled semi-homogenized laminate model is developed to predict the observed compression response of multi-directional laminates. A reduced 2-D formulation is presented first to determine the interfaces most susceptible to delamination. Subsequently, cohesive elements are added along these interfaces to introduce delamination capability in the model. Predictions of the model are compared against experimental data, and are found to be in agreement with respect to compressive strength and failure modes.
\end{abstract}

\section{Introduction}

Fiber reinforced composite laminates when subjected to compression may exhibit different types of failure mechanisms. Fiber microbuckling leading to kinking, delamination, and matrix damage are seen to dominate compressive strength. These different modes of failure occur either separately or simultaneously depending on the loading which affects the global response of the laminate. Therefore, it is of importance to study the interaction between these different types of failure mechanisms. Several previous studies have considered these failure modes to occur independently. Fiber kink banding has been identified as a compressive strength limiting mechanism in aligned fiber reinforced composite laminates by Schultheisz and Waas, ${ }^{1}{ }^{2}$ While early studies determined that the compressive strength of a single, isolated lamina can be determined by a knowledge of the lamina shear nonlinearity in the stress-strain response, in tandem with a knowledge of initial fiber misalignment, ${ }^{3},{ }^{4}$ it was later determined through a combination of experiments and numerical modeling that the kink band formation is an evolutionary process, which is governed by local stress state (including stress multi-axiality), details of the material constitutive model and the fiber misalignment angles as explained in papers by Sun and Jun, ${ }^{5}$ Kyriakides et al., ${ }^{6}$ Lee and Waas, ${ }^{7}$ Vogler et al., ${ }^{8}$ Yerramalli and Waas, ${ }^{9}$ Yerramalli and Waas, ${ }^{10}$ Basu et al., ${ }^{11}$ Pimenta et al. $,{ }^{12},{ }^{13}$ Feld et al. ${ }^{14}$ As loading proceeds, regions of fiber misalignment in the lamina undergo deformation due to combined compression and shear loading. This region is surrounded by other material whose deformation characteristics, in general, are different. The progressively increasing local fiber misalignment coupled with a softening shear nonlinearity, perpetuates a local limit-load type instability that initiates a rapid formation of a kink band. During this formation, the external tractions required to support the structure, in general, decrease, indicating an instability. The regions within the band undergo large straining while material outside the band, relax and unload. Consequently, the mechanics of this process is related to the local microstructural details, geometry and volumes of material that are occupied by the band and that are outside the band.

Lee and Waas, ${ }^{7}$ Lee et al. ${ }^{15}$ Vogler et al. ${ }^{8}$ and Pimenta et al. ${ }^{12},{ }^{13}$ have shown that kink-band formation can also involve delamination (splitting) in combination or in isolation of the band formation. Lee and Waas $^{7}$ studied the effect of fiber volume fraction on the compression failure mode, while Yerramalli and Waas $^{9}$ studied the effect of fiber type and load multi-axiality on failure. The formation of kink banding as an energy release mechanism in limiting the compressive strength of laminates with cut-outs has previously

\footnotetext{
*Graduate Student, Department of Aerospace Engineering, 1320 Beal Avenue, Ann Arbor, MI 48109-2140. AIAA Student Member.

${ }^{\dagger}$ Felix Pawlowski Professor of Aerospace Engineering, Department of Aerospace Engineering, 1320 Beal Avenue, Ann Arbor, MI 48109-2140. Fellow, AIAA.

${ }^{\ddagger}$ Comet Technology Corporation, Ann Arbor, MI 48103
} 
been addressed by Waas et al., ${ }^{16} \mathrm{Ahn}$ and Waas $,{ }^{17},{ }^{18}$ and Soutis et al., ${ }^{19}$ while the influence of fiber waviness on compression failure of unidirectional laminates has been studied by Wisnom. ${ }^{20}$ Micromechanical models to predict compressive strength using varying degrees of simplification have been proposed by Naik and Kumar, ${ }^{21}$ and, $\mathrm{Xu}$ and Reifsnider. ${ }^{22}$

While kink banding is governed by the inelastic response of the matrix material and fiber misalignment, delamination is governed by the fracture properties of the matrix and/or fiber matrix interface, ${ }^{23}$ Extension of previous findings, for a lamina, to the case of predicting the compressive strength of a multi-directional laminate is one objective of the present study. Therefore, multi-directional carbon fiber reinforced polymer (CFRP) matrix laminates are considered in this paper, with the main goal of developing a procedure to predict compressive strength and failure modes. In this regard, a semi-homogenized laminate model is constructed, where micro-mechanics is maintained in the 0 degree fibers and the off-axis layers are homogenized. Further, to predict the compressive strength and failure modes, cohesive elements are added at chosen interfaces of the model to incorporate the ability to delaminate. The weak interfaces are determined a priori by a simplified 2-D analysis of the laminate. The compressive strength and failure modes are predicted, and are compared to the experimental observations.

\section{Experiments}

Compression tests were conducted on carbon fiber reinforced polymer(CFRP) matrix laminates to understand the mechanisms of compressive failure and to examine the influence laminate thickness (scaling) on these mechanisms. The influence of two important types of failure mechanisms, namely delamination and kinking and their interaction on the compression strength is the main focus of this study. In the following sections, details about the experimental set-up, stacking sequence (also referred to as layups) of different specimens and the results of compression tests are presented.

\section{A. Test Fixture}

The Wyoming Combined Loading Compression (WCLC) test fixture shown in Fig. 1 was used to carry out the compression tests in association with a MTS loading frame. Specimens in the form of laminated strips with nominal dimensions of 0.5 inch x 5.25 inch $\mathrm{x}$ " $\mathrm{t}$ " inches, where, "t" is variable, are sandwiched between the large metal blocks of the fixture and the ends of the fixture are compressed between the flat loading platens of a MTS testing frame as shown in Fig. 1 and Fig. 2. The blocks act as anti-buckling guides during the tests, supporting a large portion of the specimen length, and providing a length of 0.25 inch as the gage length. This results in the measured compressive strength to be as close as possible to the actual compressive strength of the material, uncontaminated by any tendency towards flexural buckling.

\section{B. Specimens}

Specimens of three different layups and of varying thickness were tested under compression. These specimens are also used to study the effects of scaling, by grouping families of laminae, on the compressive strength of the laminates. As indicated in Table 1, the thicknesses of the layups are scaled up by stacking multiple layers of the same orientation. All the laminates shown in Table 1 have the same in-plane stiffnesses. All the specimens are of size 0.5 inch $x 5.25$ inch which results in a gage length of 0.25 inch when placed in the WCLC fixture as shown in Fig. 2.

Table 1. Types of Laminates

\begin{tabular}{cccc}
\hline \hline Type of Laminates & $\mathrm{L}($ in $)$ & $\mathrm{W}(\mathrm{in})$ & $\mathrm{t}(\mathrm{in})$ \\
\hline Layup 1: $[-45 /+45 / 90 / 0] \mathrm{s}$ (8 plies $)$ & 0.25 & 0.5 & 0.049 \\
Layup 2: $\left[-45_{2} /+45_{2} / 90_{2} / 0_{2}\right] \mathrm{s}(16$ plies $)$ & 0.25 & 0.5 & 0.094 \\
Layup 3: $\left[-45_{4} /+45_{4} / 90_{4} / 0_{4}\right] \mathrm{s}(32$ plies $)$ & 0.25 & 0.5 & 0.176 \\
\hline
\end{tabular}

Typical images of the laminates mentioned above are shown in Fig. 3 and Fig. 4 , where Fig. 3 displays the image of a failed Layup 1 laminate, and Fig. 4 displays a close-up image of the failed region of the 


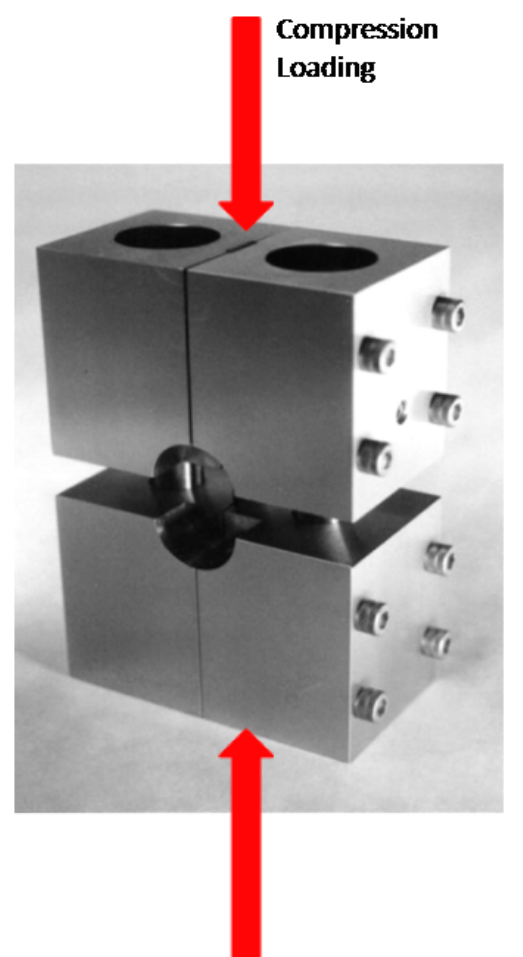

Figure 1. Assembled fixture with specimen installed

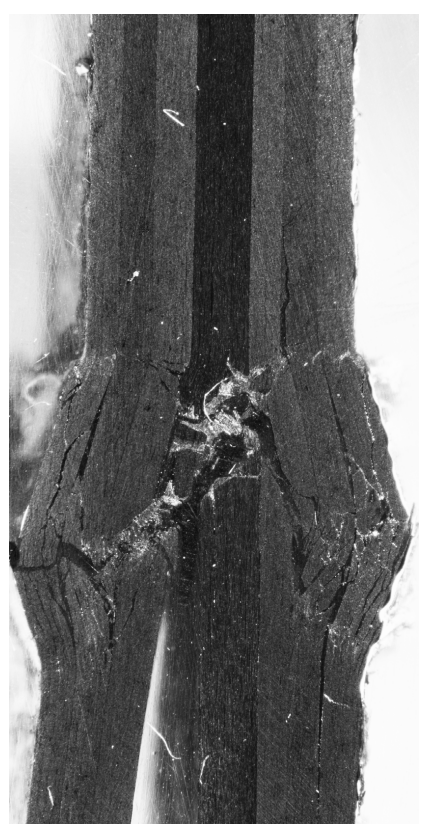

Figure 3. Failed specimen : 16 ply

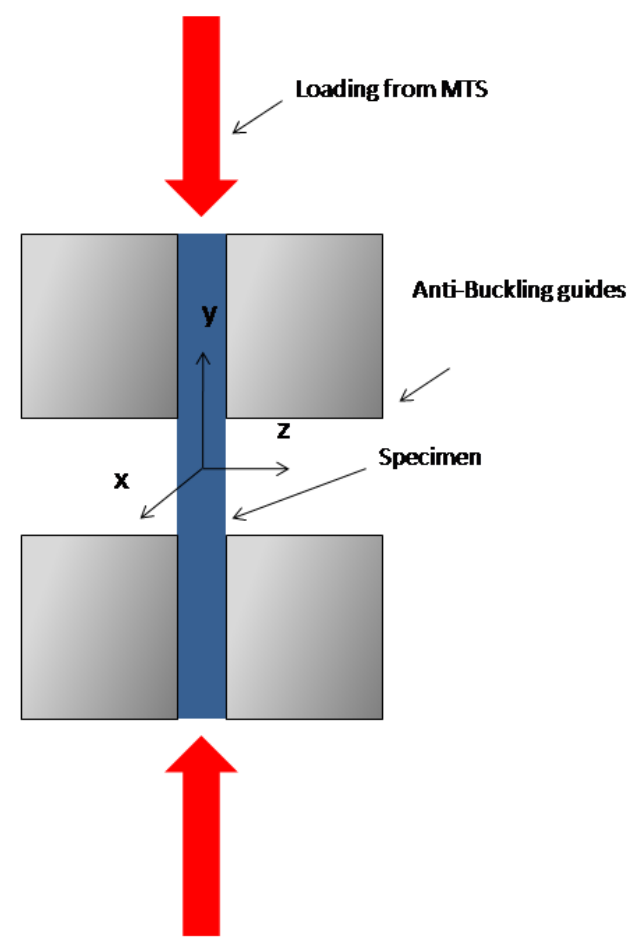

Figure 2. Sketch of loading configuration

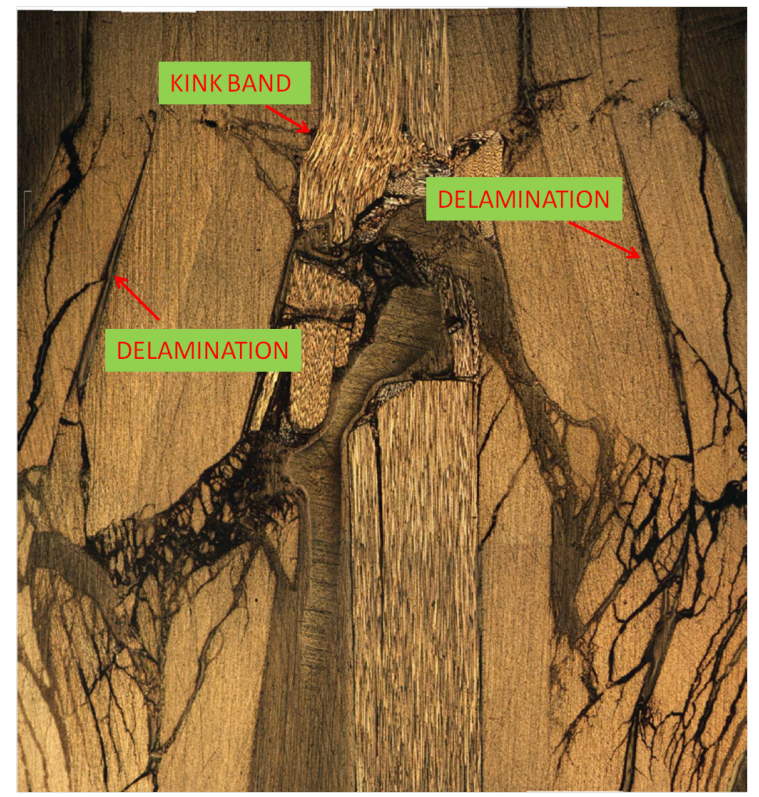

Figure 4. Detailed image of the failed region amplifying kinking and delamination zones

corresponding specimen. It is observed that the failed specimen shows delamination occurring at the interface of the laminae, and kinking in the 0 degree ply. As will be discussed later, the strain fields that persist upto and beyond failure indicate that there is a fairly uniform deformation corresponding to an initial linear relation between applied load and strain. At a critical value of the applied end displacement, a sudden transition in the stability of the specimen occurs and this leads to catastrophic failure with a significant 
reduction in the measured load.

\section{Details of the Tests}

The compression tests are carried out at an external displacement control loading rate of $0.0004 \mathrm{in} / \mathrm{sec}$ in a MTS hydraulic test frame. The "macroscopic" stress is calculated as the total load obtained from a load cell that is placed in-line with the specimen, divided by the initial undeformed cross sectional area of the specimen. The "macroscopic" strain is determined using strain gages on either faces of the specimens. The global stress-strain response for all the three specimens are shown in Fig. 5. The initial stiffness of the laminates is $48.5 \pm 0.2 \mathrm{GPa}$, and the compressive strengths are in the range of $590 \pm 30 \mathrm{MPa}$.

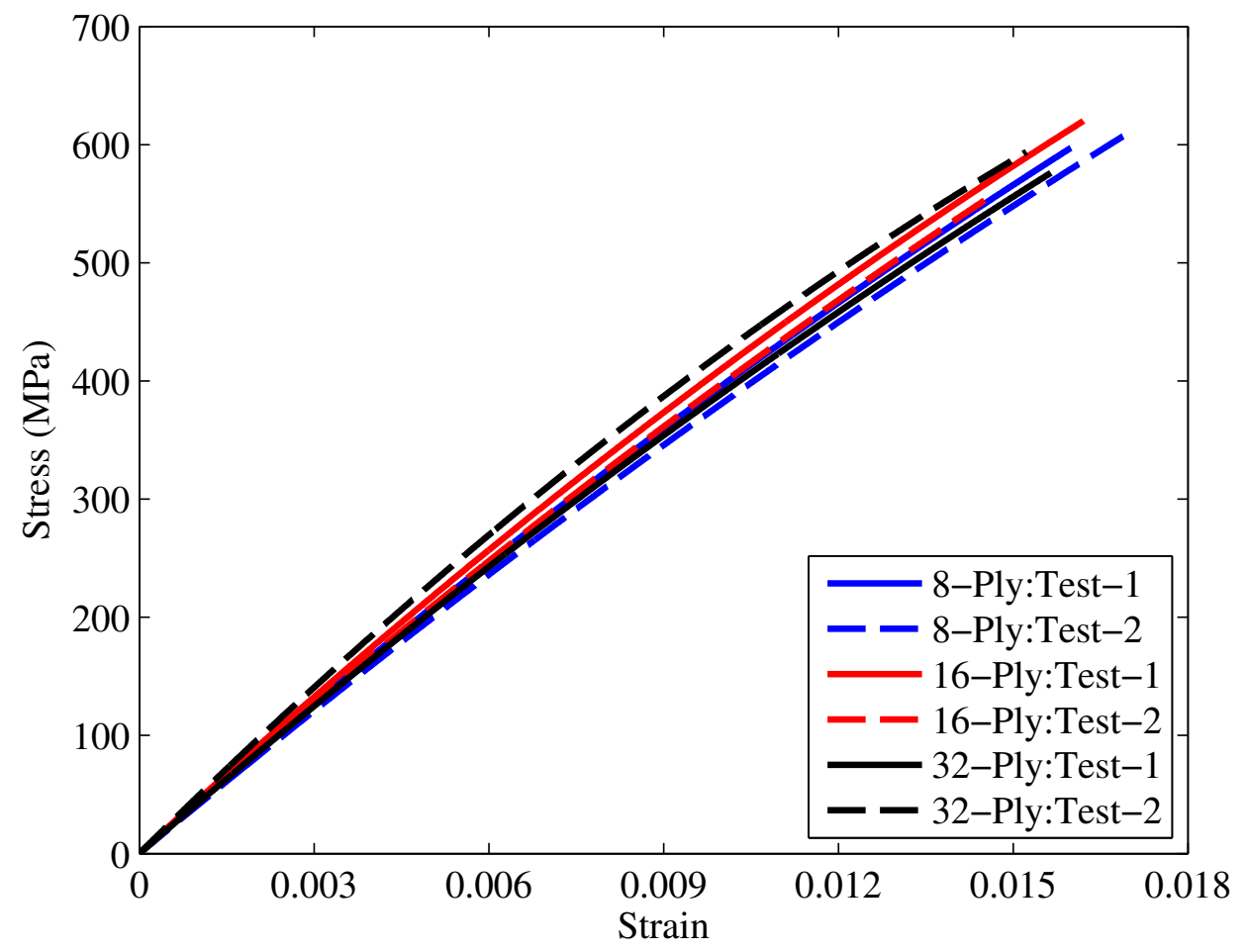

Figure 5. Global stress-strain response of the laminates determined experimentally

This implies that the scaling of laminae in the laminate has no significant influence on either the initial stiffness or the strength of the laminates.

\section{Strain Analysis of Laminates Using Digital Image Correlation (DIC) - ARAMIS}

DIC method is used to analyze strain distribution on the cross-section of the laminates. ARAMIS, a commercially available software package is used to perform the DIC analysis. ARAMIS is a non-contact and material independent displacement measuring system which gives displacements, strains and velocities as a function of time. The side surface of the specimen (through the thickness) which is to be imaged has a speckle pattern with random black dots over a white background, created using an air-brush. The side surface that is imaged is the surface with a normal in the $\mathrm{z}$ - direction, where the axes are as indicated in Fig. 2. A series of images are taken during the experiment, and these images are analyzed using ARAMIS to calculate the displacements and strains.

Fig. 6, Fig. 7 and Fig. 8 show three of the series of images of a Layup 3 specimen as a function of macroscopic stress state. The first image, a reference image that is used for the DIC calculations, corresponds to the unloaded state while the next two images correspond to an instance near the peak load and immediately thereafter. In the second image, a delamination crack is already visible and is identified as the first event that may trigger the catastrophic failure which, as seen in the third image, also induces kinking in the zero lamina in the post-peak regime. This type of failure mechanism that initiates the catastrophic failure was 


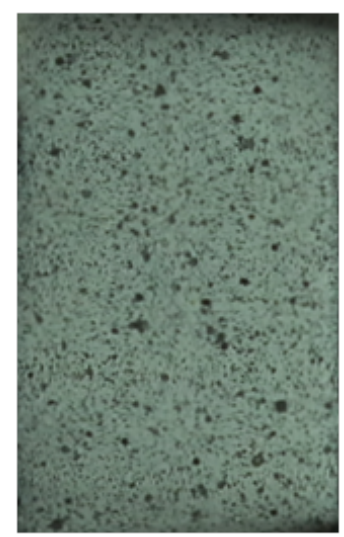

Figure 6. Unloaded

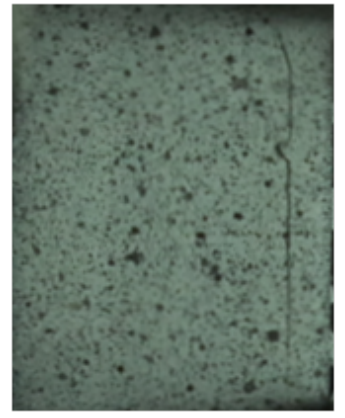

Figure 7. Peak load

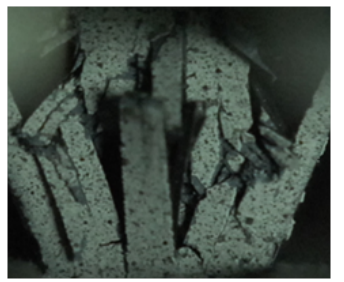

Figure 8. Failed

visible in all the laminates, regardless of the thickness scaling, and is also further supported by the DIC strain field analysis which will be discussed further below.

The DIC images of the cross-sectional area of a typical Layup 3 specimen are shown in Fig. 9a, Fig. 9b and Fig. 9c. The specimen is loaded in the global y-direction. Fig. 9a, b and c display the strain distribution on the cross-sectional area along the global x-direction. We notice that the distribution is banded along the thickness. This is due to the different layers present in the specimens. We also observe that as the loading is increased, the positive strain between the layers +45 and -45 increases rapidly, and the specimen delaminates at that interface as clearly shown in Fig. 9c. To corroborate the above statement, the strain distributions $\epsilon_{x x}$ and $\epsilon_{x y}$ along a line on the cross-section are also plotted. It is clear from Fig. 10b and Fig. 10b that as the load is increased, the transverse strain $\left(\epsilon_{x x}\right)$ and the shear strain $\left(\epsilon_{x y}\right)$ attain maximum values at the interface between +45 and -45 layers. Upon further loading, the transverse and shear strains tend to infinity as the specimen delaminates at the interface on the right (refer to Fig. 10c and Fig. 11c). In summary, the specimens appear to initiate failure by delamination followed by kink banding occurring simultaneously or in the post-peak regime.

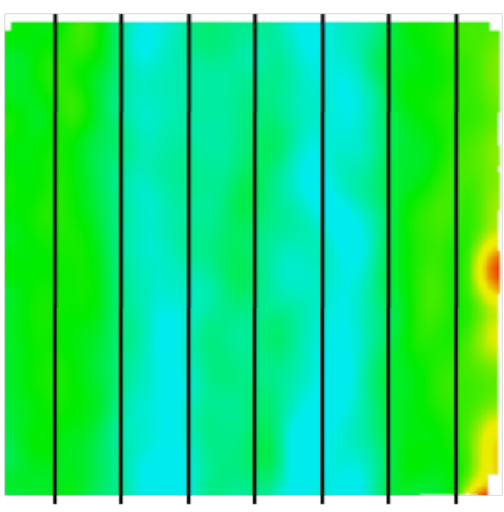

(a)

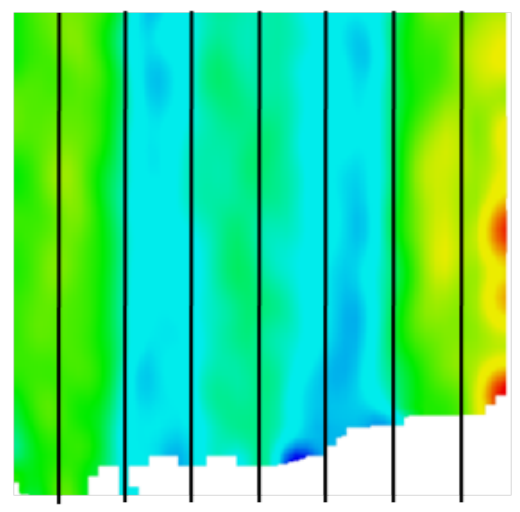

(b)

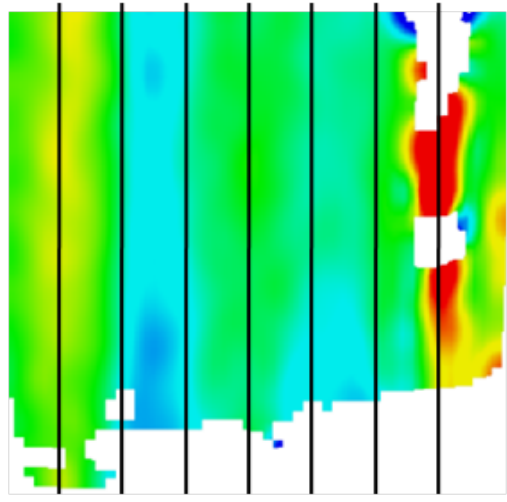

(c)

Figure 9. Transverse strain distribution on the side surface for Type-A 16-ply laminate

\section{Computational Modeling}

An upscaled homogenized laminate is constructed for Layup 1, where the stacking is $[-45 /+45 / 90 / 0] \mathrm{s}$ as explained in. ${ }^{24}$ The 0 degree laminae have hexagonal packed fibers maintaining the micro-mechanics, and the off-axis layers are homogenized using a deformation theory implementation of Hill's anisotropic plasticity theory. Interface elements formulated using the discrete cohesive zone method (DCZM), ${ }^{25}$ are added at the interface between $-45 /+45$ layers, which is determined a priori as the weakest interface in the laminate. 


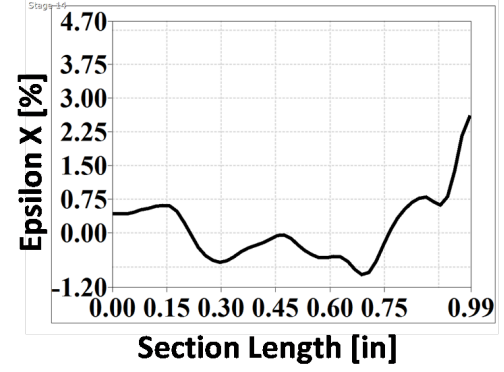

(a)

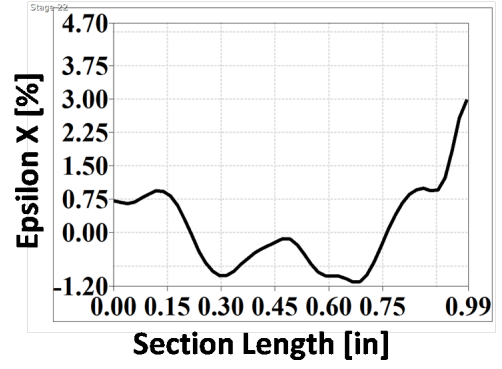

(b)

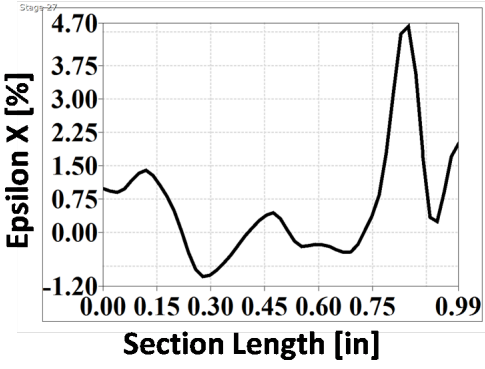

(c)

Figure 10. Transverse strain distribution across the side surface for Type-A 16-ply laminate

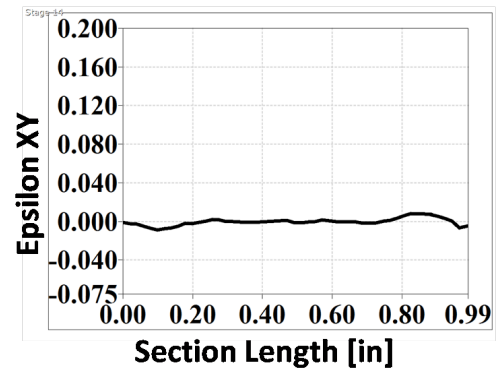

(a)

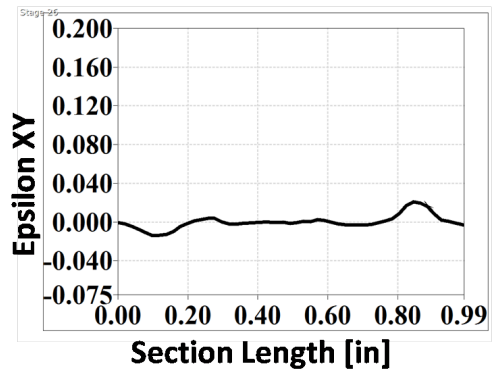

(b)

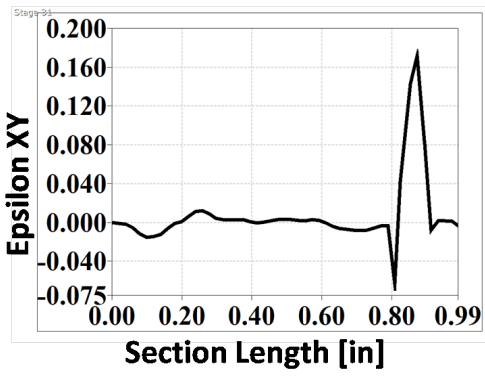

(c)

Figure 11. Shear strain distribution across the side surface

A simplified 2-D analysis of the laminate which is explained in the following sections is used to identify the interfaces most susceptible to delamination. Finally, the laminate model is compressed in the global y-direction, using displacement control, to predict the compressive strength and failure modes.

\section{A. Upscaled Laminate Model}

The upscaled homogenized model consists of 8-layers of laminae (see Fig. 12), where the off-axis layers i.e. $-45^{0}, 45^{0}, 90^{0}$ layers are homogenized using the technique mentioned in. ${ }^{24}$ Here, the red, cream and blue regions are the homogenized $-45,+45$ and 90 degree laminae. Micro-mechanics is maintained in the $0^{0}$ layers, as they are the load bearing layers and are responsible for kink band formation in multi-directional laminates. Therefore, the regions in green are the 0 degree fibers, and regions in grey are the matrix in 0 degree lamina.

The homogenized elastic lamina properties of the off-axis laminae corresponding to a volume fraction of 0.49 and material properties given in Table 2 and Fig. 13, are given in Table 3 . The constants $R_{i j}$ required to calculate the plastic potential of Hill's plasticity model are tabulated in Table 4 (note that $R_{11}$ is chosen to be an arbitrarily high value since the 1-direction of the lamina is assumed to be elastic throughout, and hence does not possess a yield strength). These properties are applied to off-axis laminae in their rotated principal material coordinate axes, accounting for different lamina orientations.

Table 2. Fiber Properties

\begin{tabular}{ccccccccc}
\hline \hline$E_{11}(\mathrm{GPa})$ & $E_{22}(\mathrm{GPa})$ & $E_{33}(\mathrm{GPa})$ & $G_{12}(\mathrm{GPa})$ & $G_{13}(\mathrm{GPa})$ & $G_{23}(\mathrm{GPa})$ & $\nu_{12}$ & $\nu_{13}$ & $\nu_{23}$ \\
\hline 276 & 8.76 & 8.76 & 12.0 & 12.0 & 3.244 & 0.35 & 0.35 & 0.35 \\
\hline
\end{tabular}

A schematic of the model shown in Fig. 14 is further used to describe the boundary conditions and loading on the model. The edge AE of the model is prevented from motion in the z-direction, and the corner $\mathrm{E}$ is fixed against moving in the global $\mathrm{x}, \mathrm{y}$ and $\mathrm{z}$-directions. The face BFGC is subjected to compression along the negative $\mathrm{x}$-direction in a displacement control manner. The faces ABCD and EFGH are held flat 


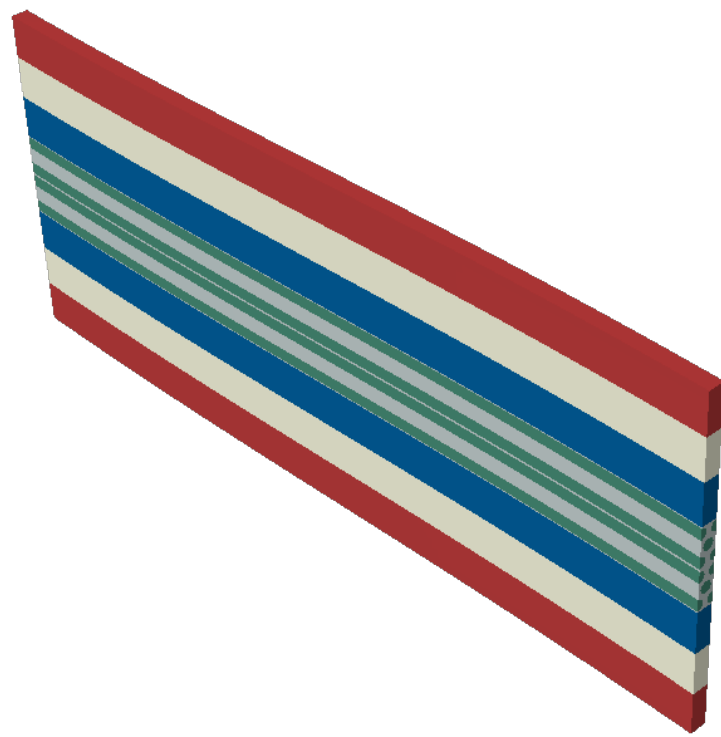

Figure 12. 8-layered upscaled model of the laminate with homogenized off-axis laminae

Table 3. Elastic Homogenized Lamina Properties

\begin{tabular}{ccccccccc}
\hline \hline$E_{11}(\mathrm{GPa})$ & $E_{22}(\mathrm{GPa})$ & $E_{33}(\mathrm{GPa})$ & $G_{12}(\mathrm{GPa})$ & $G_{13}(\mathrm{GPa})$ & $G_{23}(\mathrm{GPa})$ & $\nu_{12}$ & $\nu_{13}$ & $\nu_{23}$ \\
\hline 136.81 & 5.397 & 5.397 & 2.45 & 2.45 & 1.71 & 0.42 & 0.42 & 0.57 \\
\hline
\end{tabular}

Table 4. Values of $R_{i j}$ for calculating Hill's potential constants

\begin{tabular}{cccccc}
\hline \hline$R_{11}$ & $R_{22}$ & $R_{33}$ & $R_{12}$ & $R_{13}$ & $R_{23}$ \\
\hline 18876.5 & 1.0 & 1.0 & 0.95 & 0.95 & 1.15 \\
\hline
\end{tabular}

but are allowed to expand or contract in the y-direction. Also, the faces ABCD and EFGH deform exactly the same way in $\mathrm{x}$ and $\mathrm{z}$-directions. This enables the use of one representative unit cell in the $\mathrm{y}$-direction, along with preserving a constant initial stiffness of the laminate, regardless of the width of the model.

The Riks method option available in ABAQUS v6.10, which is an arc-length solution scheme, is adopted to conduct the compressive response analysis. As shown in previous studies $\left({ }^{26}\right)$, this method captures unstable equilibrium paths (path in the load vs. loaded edge displacement graph that show snap-back response) that can occur at limit points, as will be discussed later.

The model is meshed with 3-D hexahedral elements (C3D8 in ABAQUS v6.10). A slight imperfection is imparted to the model to account for fiber misalignment. Previous work ${ }^{27}$ has shown that initial misalignment angles of 0.5 to 2 degrees of the zero laminae bound the distribution of fiber misalignment that is typical of carbon fiber reinforced pre-preg aerospace laminates. The first buckling mode of the laminate is determined, and is used to impart an imperfection to the model as an imperfection (effective fiber misalignment) angle, $(\theta)$, where $\theta=\frac{\delta}{L}$, is defined as shown in Fig. 15 .

The initial state in the compression analysis is stress free. The model is seeded with fiber misalignment angles of $1^{0}$, and subjected to compression. Here, macroscopic stress is defined as the total resultant $\mathrm{x}$ direction reaction force on the face BFGC divided by the product of the width BF and thickness BC, while the macroscopic strain is defined as the total contraction (change in length between the faces BFGC and AEHD) divided by the initial length $\mathrm{AB}$. 


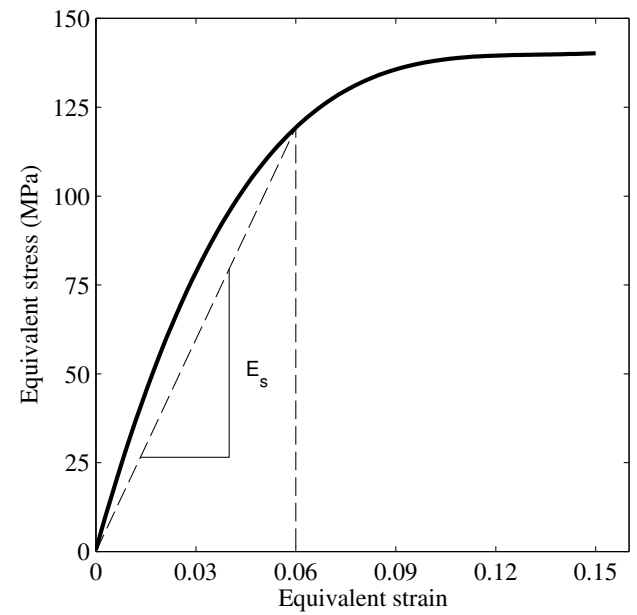

Figure 13. Equivalent stress-strain response for the in-situ matrix

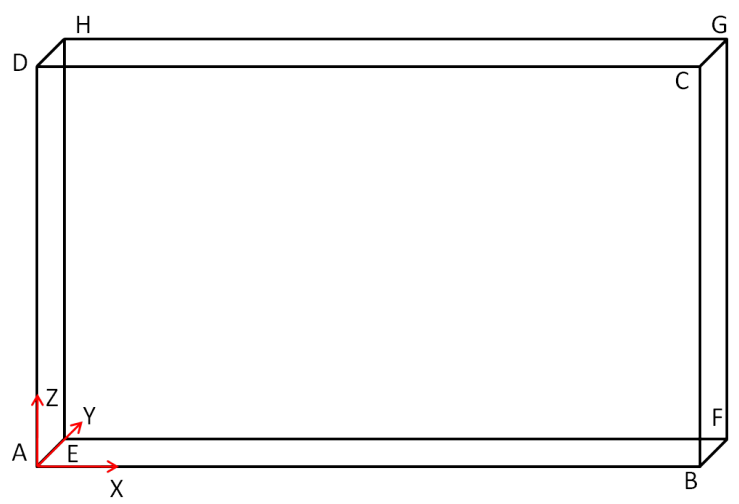

Figure 14. A schematic of the laminate model to describe boundary conditions and loading

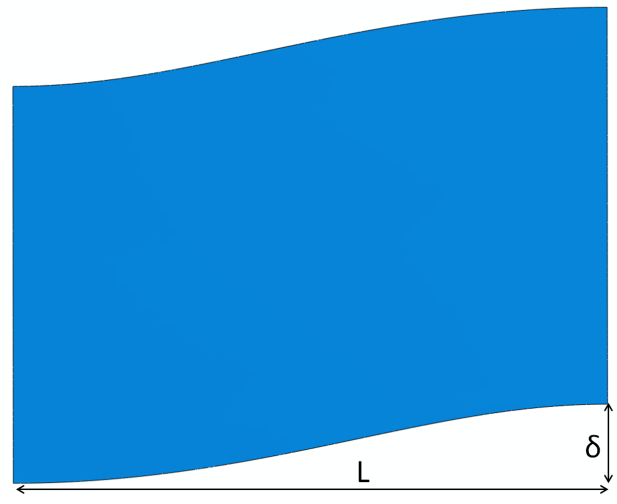

Figure 15. Laminate model to describe angle of imperfection

\section{B. 2-D reduction formulation to determine a priori weak interfaces in a laminate}

After developing the upscaled laminate model, the weakest interface in the laminate needs to be determined in order to add interface elements along that interface. In order to do that, a generalized 2-D formulation is derived as explained in the following section. 


\section{Generalized 2-D formulation}

Based on the formulation given in Pipes and Pagano, ${ }^{28}$ and Martin et al, ${ }^{29}$ a laminate of length $2 \mathrm{~L}$, width of $2 \mathrm{~b}$ and lamina thickness equal to $\mathrm{h}$ is considered. The geometry of the laminate along with its boundaries is shown in Fig. 16 The layers in the laminate are in the x1-x2 plane. A compression load is applied at the edges $\Sigma_{+L}$ and $\Sigma_{-L}$ along the x1 direction. Edges $\Sigma_{0}$ and $\Sigma_{2 b}$ are the free edges in the $\mathrm{x} 2$ direction.

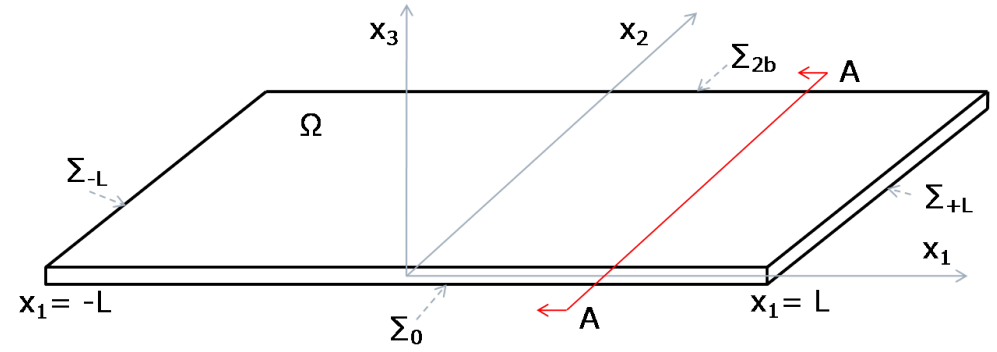

Figure 16. 3-D laminate

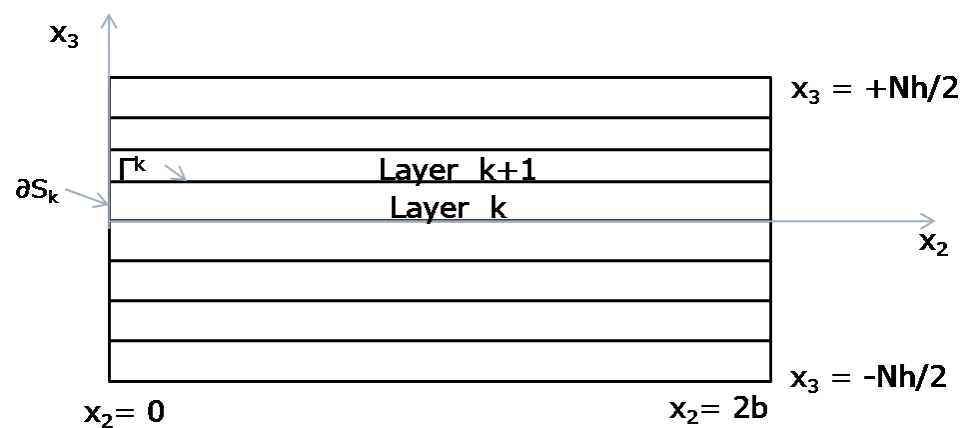

Figure 17. Cross-section of the 3-D laminate

A cross-section of the laminate at A-A is shown in Fig. 17 and has $\mathrm{N}$ layers through the thickness. The kth interface between the laminae is represented by $\Gamma^{k}$. A region considerably far from the loading surfaces is considered such that the stress components are assumed to be independent of $\mathrm{x} 1$. Then, the displacement field is given as,

$$
\begin{array}{r}
U_{1}\left(x_{1}, x_{2}, x_{3}\right)=\tilde{U}_{1}\left(x_{2}, x_{3}\right)+\epsilon_{11} x_{1} \\
U_{2}\left(x_{1}, x_{2}, x_{3}\right)=\tilde{U}_{2}\left(x_{2}, x_{3}\right) \\
U_{3}\left(x_{1}, x_{2}, x_{3}\right)=\tilde{U}_{3}\left(x_{2}, x_{3}\right)
\end{array}
$$

Here, $\epsilon_{11}$ is the applied uniform axial strain in the laminate in the x1-direction. The constitutive law for each linear elastic lamina (3-D), in tensorial form is,

$$
\sigma_{i j}=a_{i j k l} \epsilon k l
$$

where, $i, j=1,2,3$ within the laminate. Here, $a_{i j k l}$, is the fourth order linear elasticity tensor for a general anisotropic material. At the interface, we must ensure displacement and traction continuity, which are given by,

$$
\text { Displacement Continuity : }\left[U_{i}\right]=0
$$

$$
\text { Traction Continuity: }\left[\sigma_{i j} n_{j}\right]=0 \text { on the interface } \Gamma^{k}
$$

At the traction free edges we have,

$$
\sigma_{i j} n_{j}=0 \forall i=1,2,3 \text { on } \quad \Sigma_{0} \quad \text { and } \quad \Sigma_{2 b}
$$


and the boundary conditions at the loading edges are,

$$
\begin{array}{rlll}
\sigma_{i j} n_{j}=-F_{i} & \text { on } & \Sigma_{+L} \\
\sigma_{i j} n_{j}=F_{i} & \text { on } & \Sigma_{-L}
\end{array}
$$

Weak form:

Let $\mathrm{V}$ be a weighting field for the equilibrium equations, given by,

$$
V=\left(\begin{array}{l}
V_{1}\left(x_{2}, x_{3}\right) \\
V_{2}\left(x_{2}, x_{3}\right) \\
V_{3}\left(x_{2}, x_{3}\right)
\end{array}\right)
$$

The weighted average of the equilibrium equations are,

$$
\int_{\Omega} \sigma_{i j} \frac{\partial V_{i}}{\partial x_{j}}=0
$$

Since $V=f\left(x_{2}, x_{3}\right) \Rightarrow \frac{\partial V_{i}}{\partial x_{1}}=0$, which implies the weighting field is independent of $\mathrm{x} 1$.

Substituting the constitutive relation for $\sigma_{i j}$ into the above equation, we have,

$$
\int_{S} a_{i \alpha k h} \frac{\partial \tilde{U}_{k}}{\partial x_{h}} \frac{\partial V_{i}}{\partial x_{\alpha}} d x_{2} d x_{3}=-\epsilon_{11} \int_{\partial S} a_{i \alpha 11} V_{i} n_{\alpha} d S
$$

This is the generalized 2-D formulation which has 3 displacement fields but in a 2-D (x2-x3 plane) domain. The input to the above formulation is the 4th order elasticity tensor for the linear elastic material and the applied external strain .

\section{Implementation of the generalized 2-D formulation}

The above formulation can be implemented in several ways using the finite element method. The method used here is to modify a thin slice of a 3-D model to behave like a generalized 2-D model. The coordinate system used in ABAQUS is a $\mathrm{x}-\mathrm{y}-\mathrm{z}$ cartesian coordinate system which is equivalent and corresponds to the $\mathrm{x} 1-\mathrm{x} 2-\mathrm{x} 3$ coordinate system used in the previous section. A 3-D model with a small thickness in the $\mathrm{x}$-direction is considered. The model is restricted from any expansion in the $\mathrm{x}$-direction using multi-point constraints. This satisfies the requirement that the displacement fields are independent of the $\mathrm{x}$-direction. This gives us the left hand side of equation (8). The external loads given by the right hand side of equation (8) are applied to the model on the edges in the y-z plane.

\section{Determination of weak interfaces in laminates}

The current formulation is validated against two laminates with different ply stacking, and subjected to axial (x-direction) straining. The first laminate is a $\left[0^{0} / 90^{0}\right] \mathrm{s}$ laminate from Zhang et al. ${ }^{30}$ and the second is a $\left[+10^{0} /-10^{0}\right] \mathrm{s}$ laminate from Martin et al. ${ }^{29}$ The current implementation is compared against the results presented by Zhang et al. ${ }^{30}$ and Martin et al, ${ }^{29}$ and the stresses along the interfaces are found to match well. Further, the above formulation is also implemented for an 8-ply (stacking of $[-45 /+45 / 90 / 0] \mathrm{s}$ )laminate to determine the weak interfaces. Fig. 18(a) and Fig. 18(b) show strains $\epsilon_{33}, \epsilon_{13}$ and $\epsilon_{23}$ at the interface of $-45 /+45$ (Interface 1) $+45 / 90$ (Interface 2) and 90/0 (Interface 3) layers, where Fig. 18(b) displays a close up view of the strain distributions at the edges.

It is observed in Fig. 18 that $\epsilon_{13}$ is very high at Interface 1 as compared to the strains at other Interfaces. Therefore, Interface 1 is considered to be an interface that is most susceptible to delaminate. This information will be used in constructing the computational model of the laminate, i.e. cohesive elements are added along Interface 1 to allow for delamination between $-45 /+45$ layers.

\section{DCZM Elements at Interfaces}

DCZM elements are added at the interfaces of the laminate to model delamination. The DCZM elements adopt a 1D traction law capable of simulating crack formation and propagation, i.e. delamination. The 


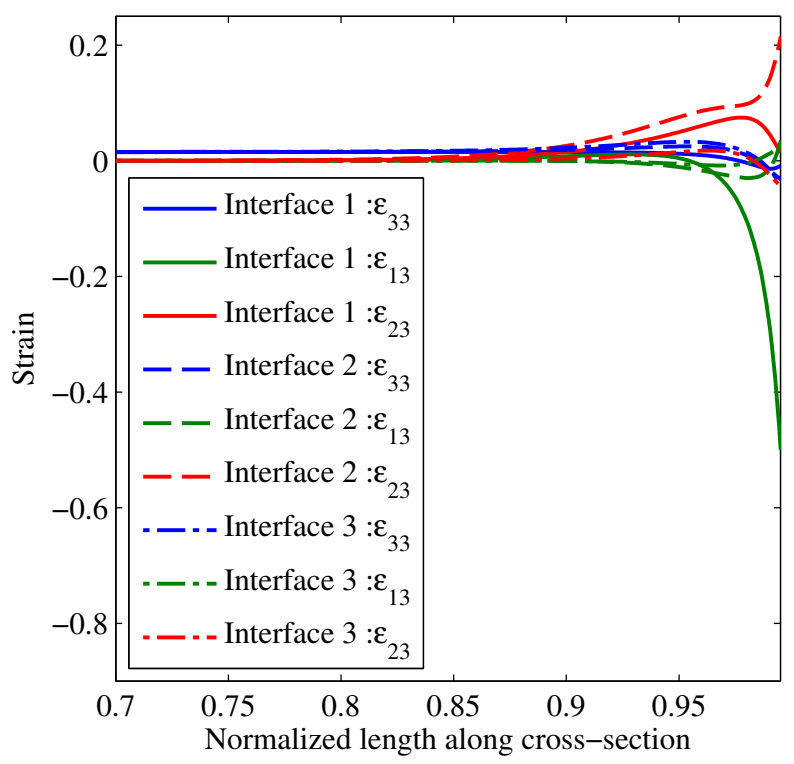

(a)

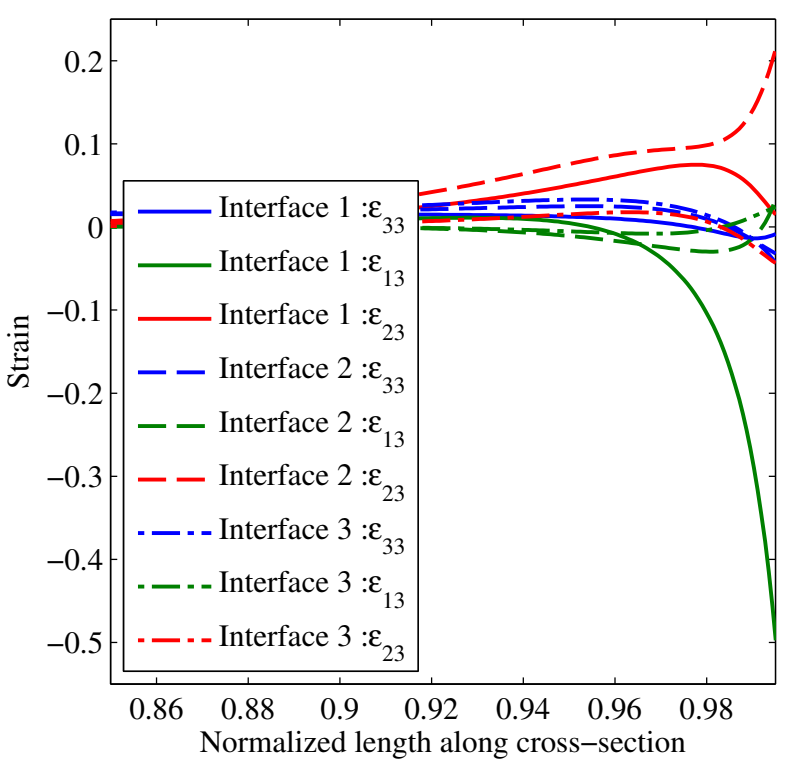

(b)

Figure 18. Strains at the interfaces of an 8-ply laminate model

element features the ability to predict delamination initiation based on a traction law that captures the cohesive strength and the fracture toughness, in each fracture mode (mode I and mode II in the current model). The DCZM elements used in this paper have been successfully employed in other studies involving crack propagation as presented in Gustafson and Waas. ${ }^{25}$ A triangular traction separation law is used here. The inputs to the law are cohesive strengths in mode-I and mode-II $\left(\sigma_{c}\right.$ and $\left.\tau_{c}\right)$, and fracture toughness in mode-I and mode-II $\left(G_{I C}\right.$ and $\left.G_{I I C}\right)$. The $G_{I C}$ and $G_{I I C}$ values are determined from the standard double cantilever beam (DCB) and edge notch flexure (ENF) tests respectively. The mode-I cohesive strength is backed out from a DCB finite element virtual test, using the experimentally determined mode-I fracture toughness as the input, and by varying the value of $\sigma_{c}$ until the load-deflection response matches the one determined experimentally. The mode-II cohesive strength is assumed to be approximately 1.5 times the mode-I cohesive strength. The fracture properties are given in Table 5. 
Table 5. Fracture Properties of Interfaces in the Laminate

\begin{tabular}{cc}
\hline \hline$G_{I C}$ & $0.7 \pm 0.07 \mathrm{~N} / \mathrm{mm}$ \\
\hline$G_{I I C}$ & $1.68 \pm 0.08 \mathrm{~N} / \mathrm{mm}$ \\
\hline$\sigma_{c}$ & $12.5 \pm 2.5 \mathrm{MPa}$ \\
\hline$\tau_{c}$ & $15 \pm 2.5 \mathrm{MPa}$ \\
\hline
\end{tabular}

\section{Laminate Model}

The upscaled homogenized model described above is implemented here with interface elements (DCZM) added along the weak interfaces which are determined a priori from the 2-D reduction analysis given above. Keeping the fracture toughnesses fixed at $0.65 \mathrm{~N} / \mathrm{mm}$ and $1.65 \mathrm{~N} / \mathrm{mm}$ in mode-I and mode-II, the cohesive strengths are varied, and the corresponding global stress-strain responses are determined. A plot of the global stress-strain responses are shown in Fig. 19.

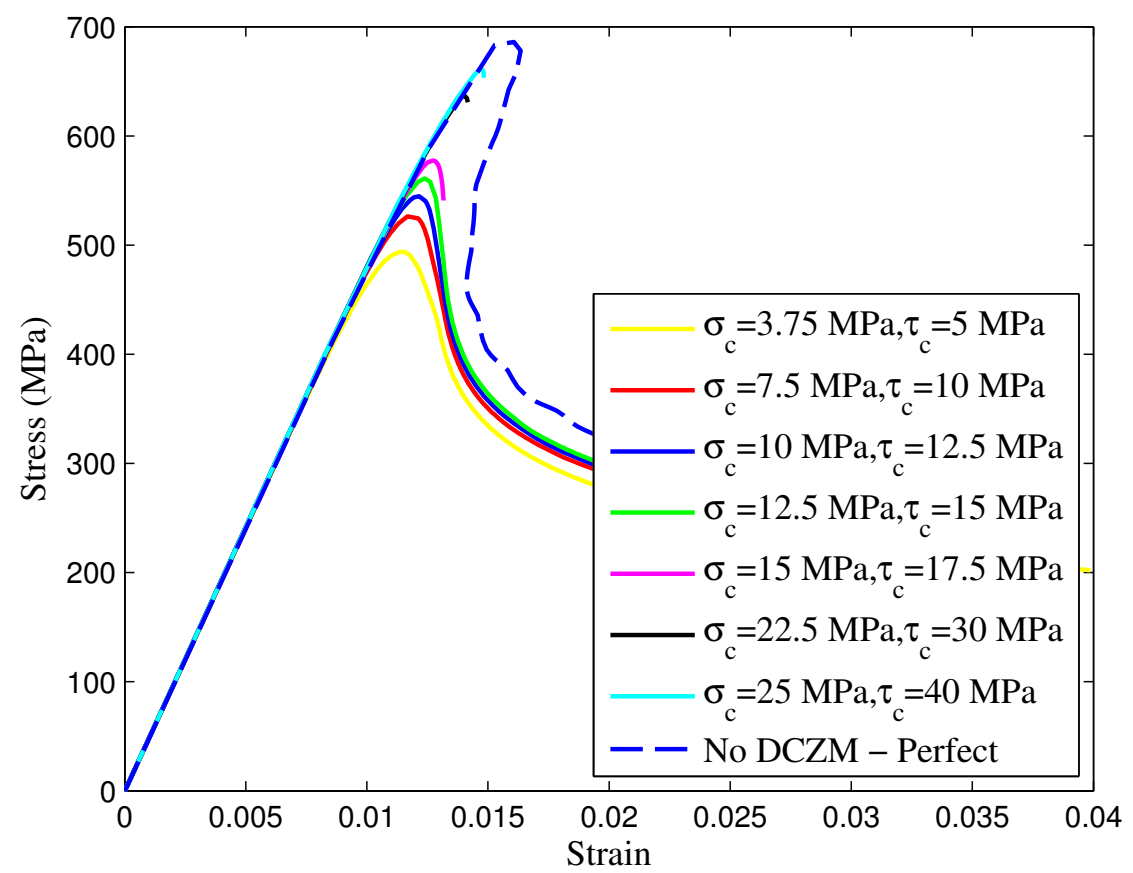

Figure 19. Global stress-strain curves of upscaled model with DCZM added at $-45 /+45$ interface

In Fig. 19, it is observed that the peak stress increases as the cohesive strengths are increased, and finally approaches the value of the model with "perfect" interfaces. For a $\sigma_{c}$ value equal to $15 \mathrm{MPa}$ and $\tau_{c}$ of 17.5 $\mathrm{MPa}$, the peak stress is $577 \mathrm{MPa}$. This is slightly lower than the compressive strength determined experimentally, as seen in Fig. 5. If the upper bound of fracture toughness values are used, i.e. $G_{I C}=$ $0.77 \mathrm{~N} / \mathrm{mm}$ and $G_{I I C}=1.76 \mathrm{~N} / \mathrm{mm}$, and $\sigma_{c}$ and $\tau_{c}$ values equal to $15 \mathrm{MPa}$ and $17.5 \mathrm{MPa}$ respectively, it is found that the peak stress is unaltered. Thus, within the bounds of the fracture toughness values obtained experimentally, and for fixed values of $\sigma_{c}$ and $\tau_{c}$, the predicted compressive strengths remains unaltered corresponding to a fiber misalignment angle of 1 degree. Other parametric studies were carried out by changing one of the fracture properties and the other three properties held fixed. It was observed that the peak stress is sensitive to the $\tau_{c}$ value. Keeping the values of $G_{I C}, G_{I I C}$ and $\sigma_{c}$ fixed at $0.77 \mathrm{~N} / \mathrm{mm}$, $1.76 \mathrm{~N} / \mathrm{mm}$ and $15 \mathrm{MPa}$ respectively, the value of $\tau_{c}$ was varied between $17.5 \mathrm{MPa}$ and $30 \mathrm{MPa}$, and the peak stress was observed to increase from $577 \mathrm{MPa}$ to $620 \mathrm{MPa}$. Therefore, $\tau_{c}$ is an important factor in influencing the compressive strength of multi-directional laminates, and a good estimate of $\tau_{c}$ will provide an improved prediction of the compressive strength of these laminates. An asymmetric double edge notched 


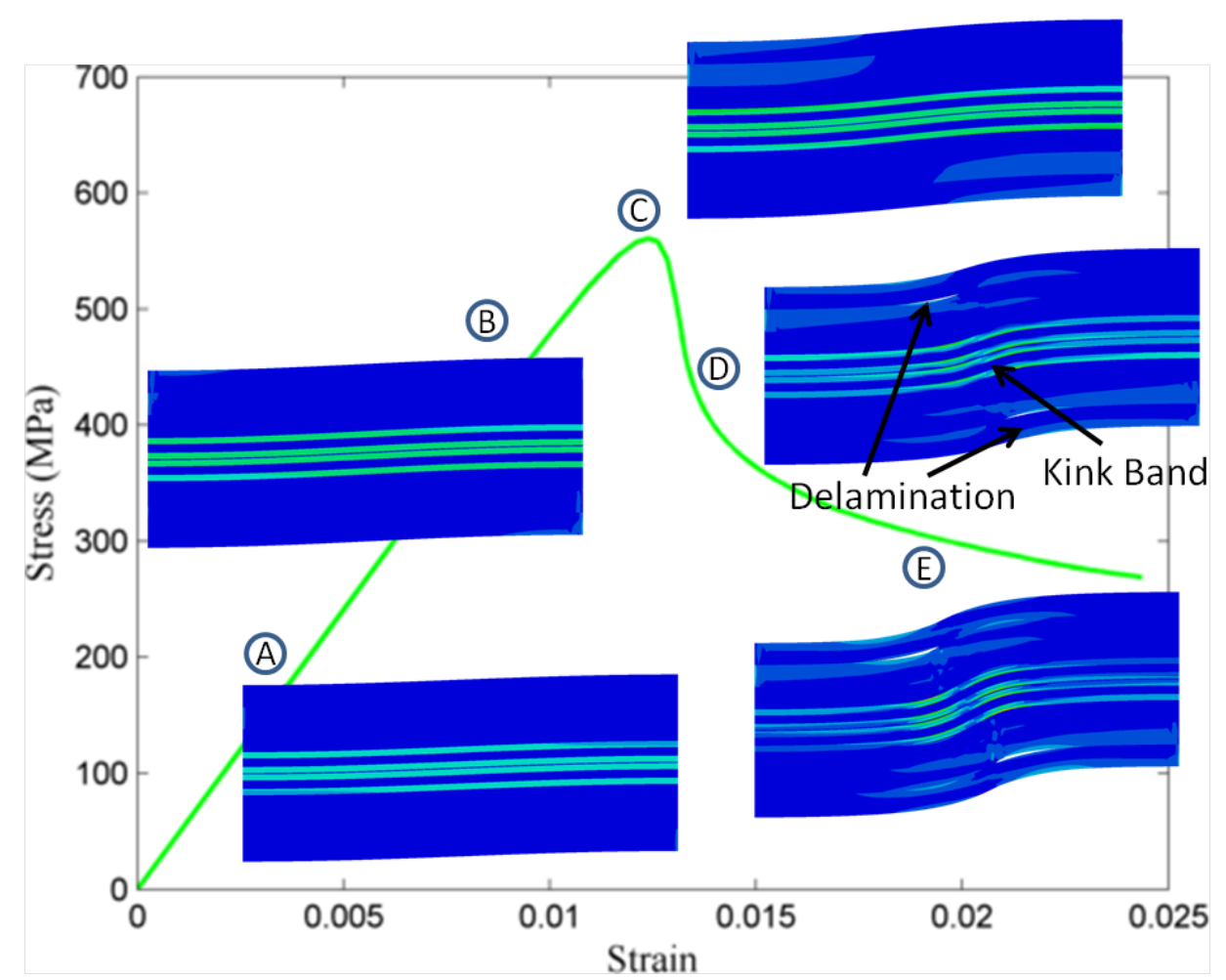

Figure 20. Global stress-strain curves along with deformed shapes of upscaled model with DCZM added at $-45 /+45$ interface. $\sigma_{c}=12.5 \mathrm{MPa}$ and $\tau_{c}=15 \mathrm{MPa}$

tension specimen is currently being implemented to measure $\tau_{c}$ from the same batch of material as that used for the compression experiments.

The stress-strain response corresponding to $G_{I C}=0.65 \mathrm{~N} / \mathrm{mm}, G_{I I C}=1.65 \mathrm{~N} / \mathrm{mm}, \sigma_{c}=12.5 \mathrm{MPa}$ and $\tau_{c}=15 \mathrm{MPa}$ (mean values of cohesive strengths), along with deformed shapes of the model are shown in Fig. 20. Here, at the peak i.e. at loading step corresponding to C, sliding between the interfaces of -45 and +45 layers is observed, and in the unloading region i.e. loading step $\mathrm{D}$, formation of a kink band is observed along with delamination at the $-45 /+45$ interface. This implies that the failure strength is influenced by fracture properties of the laminate, and so is the failure mode. Here, a combination of kinking and delamination is observed in the post-peak regime, with the compressive strength affected by cohesive strengths of the delaminating interfaces. Further studies will examine the effects of stacking, and the effects of relative magnitudes between fracture properties and matrix shear non-linearity in influencing the compressive strength and associated failure mode/s of laminates.

\section{Conclusions}

A computational model to predict compressive strength of CFRP laminates and associated failure modes has been presented. The model facilitates delamination to take place along the weakest interface by adding cohesive DCZM interface elements along selected "weak" interfaces. These interfaces are determined a-priori from a knowledge of laminate stacking sequence and layer geometry. The properties of the DCZM elements are based on interlaminar fracture properties determined separately. The predicted compressive strengths, when compared against a set of available experimental results, are found to agree well, both in terms of maximum load and failure mode. The mode of failure is determined by the number and orientation of the laminae, the material shear nonlinearity (dictated by the matrix properties) and the interlaminar fracture properties. Clearly, the relative magnitudes of the fracture parameters and a measure of the lamina material nonlinearity, captured by a non-dimensional parameter, much like that presented in $^{23}$ for a single lamina, is needed for a multi-directional laminate to a-priori determine the mode of failure. However, the methodology 
that is outlined in this paper can be used to quickly assess the compressive strength of laminates with a knowledge of fundamental material properties as inputs.

\section{Acknowledgements}

We are grateful for financial sponsorship from the AFOSR through a STTR grant to Comet Technology Corporation and the University of Michigan, with Dr. Ed Forster, AFRL and Dr. David Stargel as project monitors.

\section{References}

${ }^{1}$ Waas, A. and Schultheisz, C., "Compressive failure of composites .1. Testing and micromechanical theories," Progress in Aerospace Sciences, Vol. 32, No. 1, 1996.

${ }^{2}$ Waas, A. and Schultheisz, C., "Compressive failure of composites .2. Experimental studies," Progress in Aerospace Sciences, Vol. 32, No. 1, 1996.

${ }^{3}$ Budiansky, B. and Fleck, N., "Compressive failure of fiber composites," Journal of the Mechanics and Physics of Solids, Vol. 41, No. 1, 1993, pp. 183211.

${ }^{4}$ Schapery, R., "Prediction of compressive strength and kink bands in composites using a work potential," International Journal of Solids and Structures, Vol. 32, No. 6-7, 1995, pp. 739765.

${ }^{5}$ Sun, C. and Jun, A., "Compressive strength of unidirectional fiber composites with matrix non-linearity," Composites Science and Technology, Vol. 52, No. 4, 1994.

${ }^{6}$ Kyriakides, S., Arseculeratne, R., Perry, E., and Liechti, K., "On the compressive failure of fiber-reinforced composites," International Journal of Solids and Structures, Vol. 32, No. 6-7, 1995.

${ }^{7}$ Lee, S. and Waas, A., "Compressive response and failure of fiber reinforced unidirectional composites," International Journal of Fracture, Vol. 100, 1999.

${ }^{8}$ Vogler, T., Hsu, S.-Y., and Kyriakides, S., "On the initiation and growth of kink bands in fiber composites. Part II: analysis," International Journal of Solids and Structures, Vol. 38, 2001, pp. 2653-2682.

${ }^{9}$ Yerramalli, C. and Waas, A., "A failure criterion for fiber reinforced polymer composites under combined compressiontorsion loading," International Journal of Solids and Structures, Vol. 40, No. 5, 2003.

${ }^{10}$ Yerramalli, C. and Waas, A., "The effect of fiber diameter on the compressive strength of composites - A 3D finite element based study," Computer Modeling in Engineering and Sciences, Vol. 6, No. 1, 2004.

${ }^{11}$ Basu, S., Waas, A., and Ambur, D., "Compressive failure of fiber composites under multi-axial loading," Journal of the Mechanics and Physics of Solids, Vol. 54, No. 3, 2006.

${ }^{12}$ Pimenta, S., Gutkin, R., Pinho, S., and Robinson, P., "A micromechanical model for kink-band formation: Part I Experimental study and numerical modelling," Composites Science and Technology, Vol. 69, 2009.

${ }^{13}$ Pimenta, S., Gutkin, R., Pinho, S., and Robinson, P., "A micromechanical model for kink-band formation: Part IIAnalytical modelling," Composites Science and Technology, Vol. 69, 2009.

${ }^{14}$ Feld, N., Allix, O., and Baranger, E., "Micro-mechanical prediction of UD laminates behavior under combined compression up to failure: influence of matrix degradation," Journal of Composite Materials, Vol. 45, No. 22, 2011.

${ }^{15}$ Lee, S., Yerramalli, C., and Waas, A., "Compressive splitting response of glass-fiber reinforced unidirectional composites," Composites Science and Technology, Vol. 60, 2000.

${ }^{16}$ Waas, A., Babcock Jr., C., and Knauss, W., "An experimental study of compression failure of fibrous laminated composites in the presence of stress gradients," International Journal of Solids and Structures, Vol. 26, No. 9-10, 1990.

${ }^{17} \mathrm{Ahn}, \mathrm{J}$. and Waas, A., "A micromechanics-based finite element model for compressive failure of notched uniply composite laminates under remote biaxial loads," Journal of Engineering Materials and Technology - Transactions of the ASME, Vol. 121, No. 3, 1999.

${ }^{18} \mathrm{Ahn}$, J. and Waas, A., "Prediction of compressive failure in laminated composites at room and elevated temperature," AIAA Journal, Vol. 40, No. 2, 2002.

${ }^{19}$ Berbinau, P., Soutis, C., and Guz, I., "Compressive failure of $0^{0}$ unidirectional carbon-fibre-reinforced plastic (CFRP) laminates by fibre microbuckling," Composites Science and Technology, Vol. 59, 1999, pp. 1451-1455.

${ }^{20}$ Wisnom, M., "The Effect of Fibre Waviness on the Relationship between Compressive and Flexural Strengths of Unidirectional Composites," Journal of Composite Materials, Vol. 28, 1994.

${ }^{21}$ Naik, N. and Rajesh, S., "Compressive strength of unidirectional composites: evaluation and comparison of prediction models," Composite Structures, Vol. 46, No. 3, 1999.

${ }^{22} \mathrm{Xu}$, Y. and Reifsnider, K., "Micromechanical Modeling of Composite Compressive Strength," Journal of Composite Materials, Vol. 27, 1993.

${ }^{23}$ Yerramalli, C. and Waas, A., "A nondimensional number to classify composite compressive failure," Journal of Applied Mechanics - Transactions of the ASME, Vol. 71, No. 3, 2004.

${ }^{24}$ Prabhakar, P. and Waas, A., "Upscaling from Micromechanics Model to Capture Laminate Compressive Strength due to Kink Banding Instability," Computational Material Science, 2012 (Under Review).

${ }^{25}$ Gustafson, P. and Waas, A., "The influence of adhesive constitutive parameters in cohesive zone finite element models of adhesively bonded joints," International Journal of Solids and Structures, Vol. 46, No. 10, 2009, pp. 2201-2215. 
${ }^{26}$ Beghini, A., Bazant, Z., Waas, A., and Basu, S., "Postcritical imperfection sensitivity of sandwich or homogenized orthotropic columns soft in shear and in transverse deformation," International Journal of Solids and Structures, Vol. 43, No. 18-19, 2006.

${ }^{27}$ Yurgartis, S., "Measurement of small angle fiber misalignments in continuous fiber composites," Composites Science and Technology, Vol. 30, 1987, pp. 279-293.

${ }^{28}$ Pipes, R. and Pagano, N., "Interlaminar Stresses in Concrete in Stresses in Composite Laminates under Uniform Axial Extension," Journal of Composite Materials, Vol. 4, 1970.

${ }^{29}$ Martin E., Leguillon, D. and Carrre, N., "A twofold strength and toughness criterion for the onset of free-edge shear delamination in angle-ply laminates," International Journal of Solids and Structures, Vol. 47, 2012, pp. 1297-1305.

${ }^{30}$ Zhang, D., Y. J. and Sheng, H., "Free-edge and ply cracking effect in cross-ply laminated composites under uniform extension and thermal loading," Composite Structures, Vol. 76, 2006. 